Rev. Fac. Med. vet. Zootec. Univ. S. Paulo

$12: 127-32,1975$

\title{
ASPECTOS RADIOGRÁFICOS DE BEZOARES DE RUMINANTES. APRESENTAÇÃO DE CASO CLINICO EM CABRA (Capra hircus)
}

\author{
Benedicto Wlademir DE MARTIN * \\ Eduardo H. BIRGEL ** \\ Leonardo M. ARAUJO ** \\ Masao IWASAKI ***
}

\begin{abstract}
\begin{tabular}{l|l|}
\cline { 2 - 2 } RF-A/12 \\
\hline
\end{tabular}
De Martin, B. W.; Birgel, E. H.; Araujo, L. M.; Iwasaki, M. - Aspectos radiográficos de bezoares de ruminantes. Apresentação de caso clínico em cabra (capra hircus). Rev. Fac. Med. vet. Zootec. Univ. S. Paulo, 12:127-132, 1975.

RESUM0: $\mathrm{O}_{8}$ Bezoares foram clinica e radiograficamente detectados em cabras de onze anos. Os Bezoares obtidos de outros animais foram estudados radiograficamente e os resultados comparados com o caso clínico aqui descrito. A possibilidade da gênese destas estruturas é discutida neste trabalho.

UNITERMOS: Radiologia*; Bezoares*; Ruminantes*; Caprinos.
\end{abstract}

I N T R O D U C A O

Os bezoares ou egagrópilos são concreções sólidas observados com freqüência nos compartimentos gástricos ou intestinos dos ruminantes. Estas formaçōes que se originam em núcleo de constituição variável, sobre o qual se depositam substâncias orgânicas ou minerais, estão relacionados a fatores alimentares e distúrbios da nutrição.
Os egagrópilos não são facilmente evidenciados por meios semiológicos rotineiros, embora possam ser responsabilizados por síndrome de obstrução.

A gênese e ocorrência destas formaçōes em ruminantes foram estudados principalmente por BERGUI ${ }^{1}$ (1956), FERRARI ${ }^{2}$ (1957), ZANNELLI \& ZIMBARO ${ }^{6}$ (1961),

* Prof. Livre-Docente. Departamento de Cirurgia e Obstetrícia da Faculdade de Medicina Veterinária e Zootecnia da U.S.P.

** Prof. Livre-Docente.

Departamento de Patologia e Clínica Médicas da Faculdade de Medicina Veterinária e Zootecnia da U.S.P.

*** Prof. Assistente. Departamento de Cirurgia e Obstetrícia da Faculdade de Medicina Veterinária e Zootecnia da U.S.P. 
DE MARTIN, B. W.; BIRGEL, E. H.; ARAUJO, L. M.; IWASAKI, M. - Aspectos radiograficos de bezoares de ruminantes. Apresentação de caso clínico em cabra (capra hircus). Rov. Fac. Mod. vet. Zootec. Univ. S. Paulo,12:127-32, 1975.

HUTYRA et al. ${ }^{3}$ (1968), JUBB \& $\mathrm{KEN}$ NEDY $^{4}$ (1974) e SEREN 5 (1975).

Objetívamos, neste estudo, mostrar imagens radiográficas dos bezoares e demonstrar a possibilidade de evidenciá-los nos reservatãrios gástricos dos pequenos ruminantes mediante auxílio de exames com raios $\mathbf{X}$.

\section{MATERIAL E MÉTODOS}

Foram estudados através de radiografias 14 bezoares coletados de bovinos e caprinos, classificados genericamente como tricofitobezoares ou mistos e, um caso clínico deste processo em caprino.

As radiografias foram efetuadas com aparelho Tridoros 4, de $1000 \mathrm{~mA}$ e a quilovoltagem e miliamperagem foram calculadas a partir da espessura da peça ou do animal.

\section{APRESENTAÇÃO DO CASO CLANICO!}

Durante o exame clínico de uma cabra mestiça, de aproximadamente 11 anos de idade, observou-se a presença de formações globosas, de consistência dura no interior do rume. Outra formação, semelhante, porém sem a mesma mobilidade era palpada na região lombar direita.

As radiografias simples executadas em posições ventro-dorsal e lateral direita, mostraram a presença de 3 formações esféricas localizadas nos limites do rume, onde evidenciava-se, também, um dos rins aumentado de volume, com alteração n̊ densidade radiográfica da pelve (Fig. 1). A urografia excretora executada com auxílio de compostos triiodados hidrossolú veis*, demonstrou que o rim esquerdo (embora eliminando normalmente o contraste) estava aumentado de volume (Fig. 2).

Após a morte do animal, constatou-se que as referidas formaçōes esféricas eram bezoares.

\section{R E S U L T A D O S}

As radiografias dos bezoares isolados demonstraram que os núcleos são radiotransparentes (Fig. 3-A, 4-A, B, C, D e E) ou radiopacos (Fig. 3-B e 4-F).

Os aspectos radiográficos destas formações permitiram concluir pela semelhança daqueles encontrados no caso clínico aqui descrito.

Pela urografia excretora pode-se perceber que uma das formacōes palpadas tratava-se do rim hipertróflco.

\section{I S C U S S A O}

A evidência dos bezoares encontrados no caso clínico aqui descrito, levou-nos a radiografar uma série destas formações colhidas de bovinos e caprinos, com a finalidade de estudar suas imagens.

Com isto, verificou-se que conforme a opinião de alguns autores $1,2,3,4$ e 5 pode-se notar a presença de núcleos radiopacos ou radiotransparentes, na dependência da rausa que teria desencadeado a formação do núcleo. Chamou-nos atenção que alguns bezoares não mostraram impregnação por substâncias radiopascas, por tratar-se de tricobezoares (Figs. 3-A e 4-A). Outros cuja gênese do núcleo era de aspecto radiotransparente apresentava a seguir áreas concêntricas de minerais (Fig. 4-B, C, D e E). Os bezoares de núcleo radiopaco (Figs. 3-B e 4-F) mostram origens diferentes uma vez que o núcleo de um deles desenvolveu-se a partir de fragmento metálico.

Estas observações conflrmam a opinião dos autores de que a sua formação está na dependência de fatores alimentares ou distúrbios da nutriçāo e sua evoluçāo independente da continuidade ou não do agente causal.

Os bezoares nos pequenos ruminantes são passíveis de evidenciação clínica à palpação, entretanto a inspeção radiográfica, mostra imagem característica, principalmente se estiverem impregnados por minerais, permitindo, até mesmo indicar sua origem.

* Hypaque 60\% - Winthrop Products Inc. 
DE MARTIN, B. W.; BIRGEL, E. H.; ARAUJO, L. M.; IWASAKI, M. - Aspectos radiográficos de bezoares de ruminantes. Apresentação de caso clínico em cabra (capra hircus). Rev. Fac. Med. vet. Zootec. Univ. S. Paulo,12:127-32, 1975.

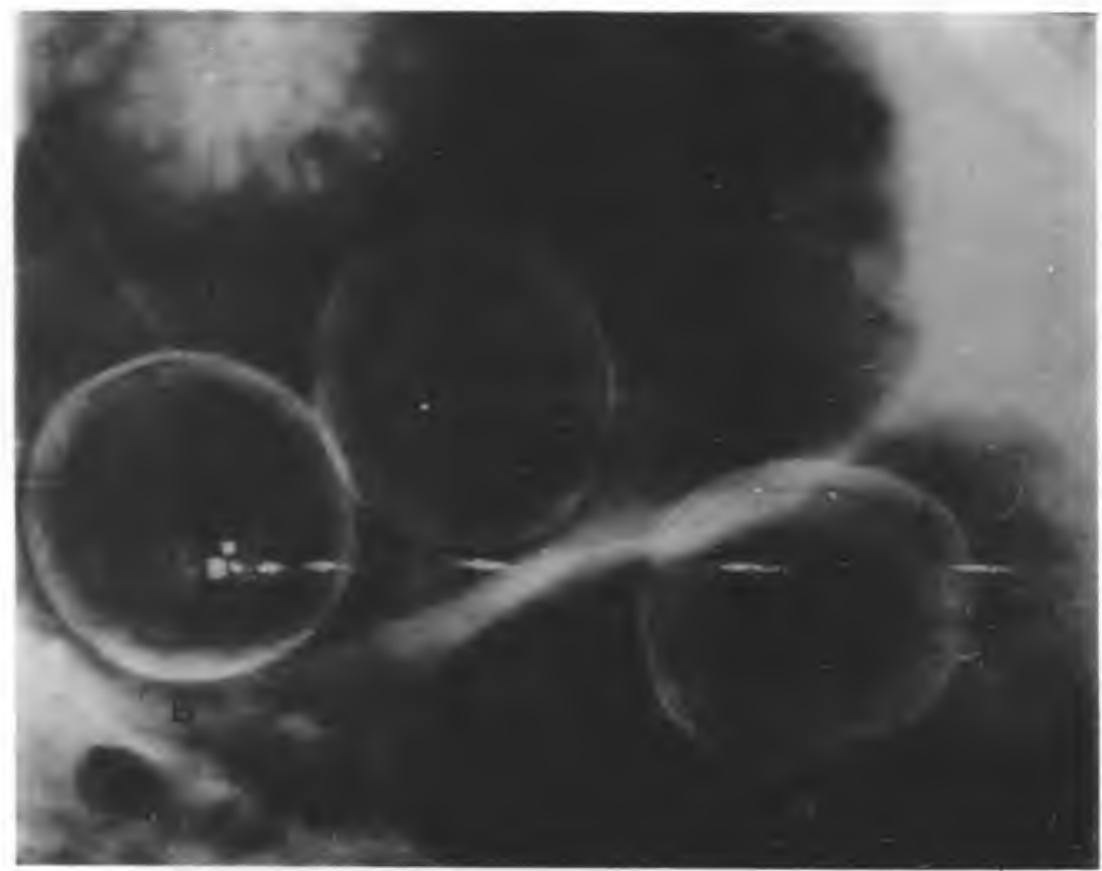

Fig. 1 - Radiografia simples, lateral mostrando os bezoares (B) e o rim esquerdo (RE) com densidade da pelve aumentada. 
DE MARTIN, B. W.; BIRGEL, E. H.; ARAUJO, L. M.; IWASAKI, M. - Aspectos radiográficos de bezoares de ruminantes. Apresentação de caso clínico em cabra (capra hircus). Rev. Fac. Med. vet. Zootec. Univ. S. Paulo,12:127-32, 1975.

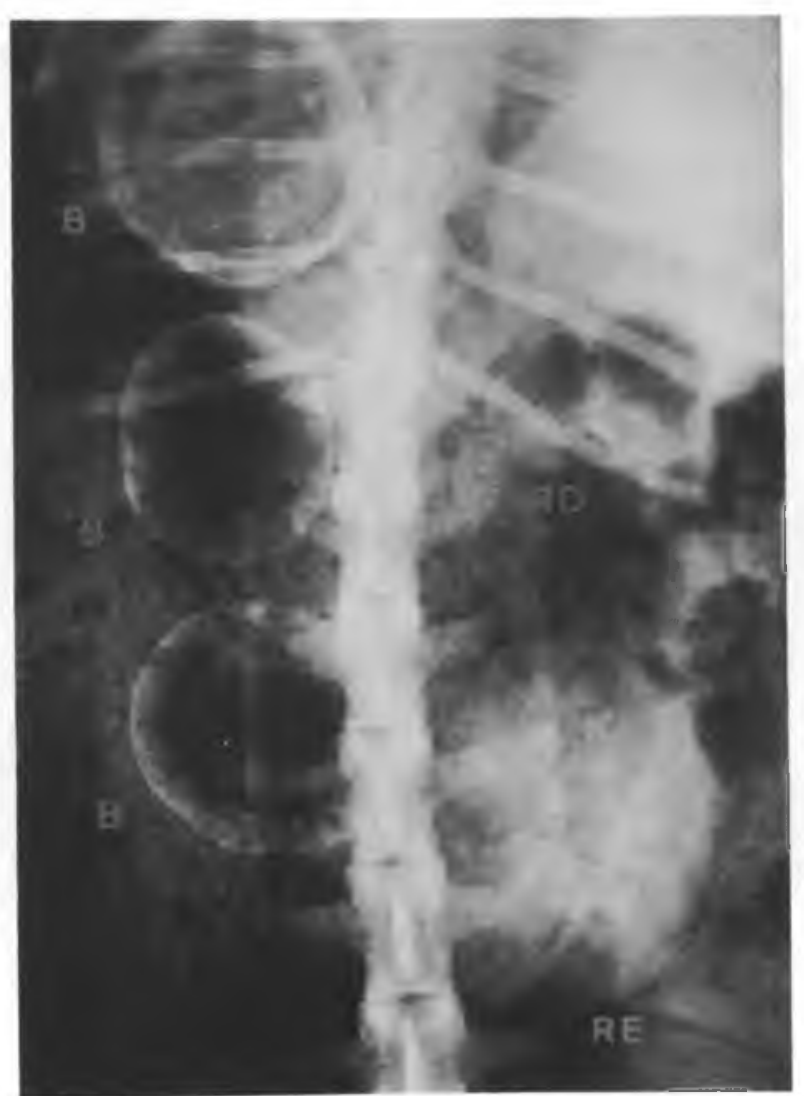

Fig. 2 - Radiografia ventro-dorsal, tomada aos 15 minutos após a administraçāo do contraste mostrando o rim esquerdo hipertrofiado (RE) e os bezoares (B) colocados à esquerda. 
DE MARTIN, B. W.; BIRGEL, E. H.; ARAUJO, L. M.; IWASAKI, M. - Aspectos radiográfico de bezoares de ruminantes. Apresentação de caso clínico em cabra (capra hirous). Rev. Fac. Med. vet. Zootec. Univ. S. Paulo, 12:127-32, 1975.

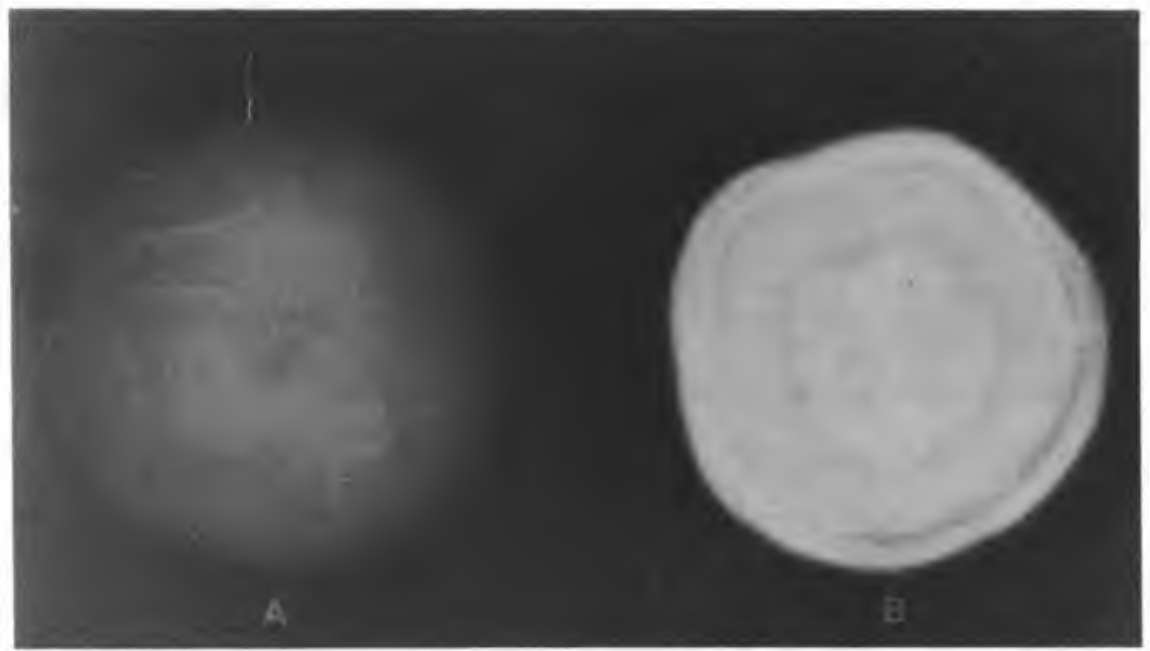

Fig. 3 - Imagem radiografica de tricobezoar puro (A) e de tricofitobezoar desenvolvido a partir de fragmento metálico (B).

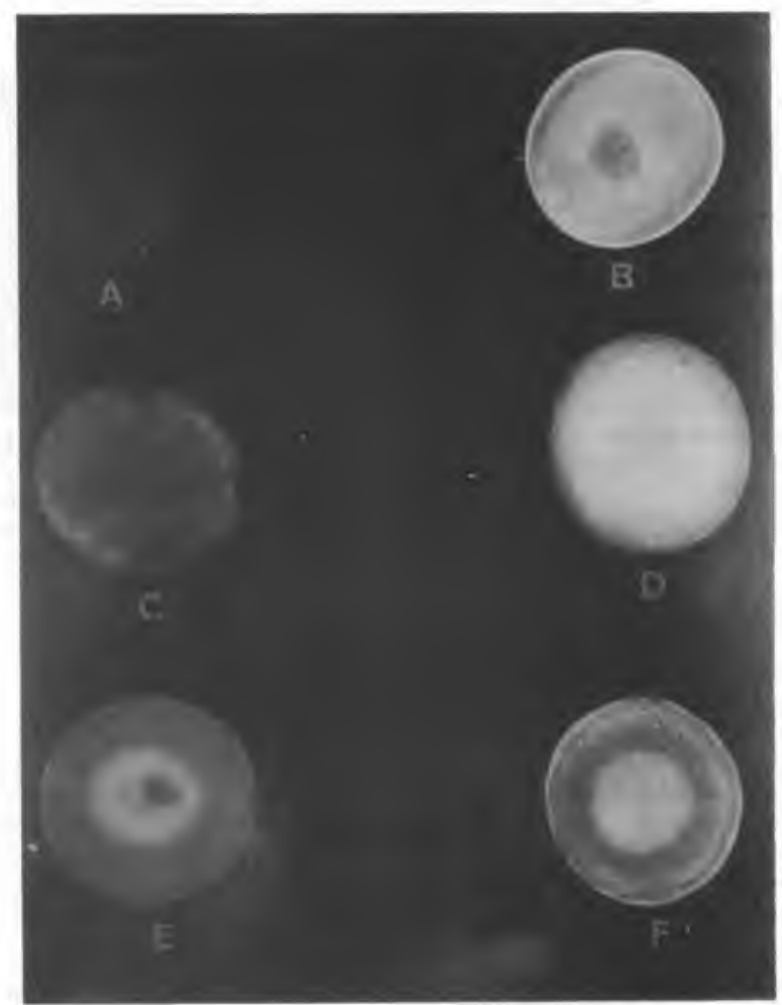

Fig. 4 - Imagens radiográficas de bezoares de ruminantes mostrando a origem orgânica ( $A, B, C, D$ e E) ou mineral (F) do núcleo. 
DE MARTIN, B. W.; BIRGEL, E. H.; ARAUJO, L. M.; IWASAKI, M. - Aspectos radiográficos de bezoares de ruminantes. Apresentação de caso clínico em cabra (capra hircus). Rev. Fac. Med. vet. Zootec. Univ. S. Paulo,12:127-32, 1975.

RFMV-A/12

De Martin, B. W.; Birger, E. H.; Araujo, L. M.; Iwasaki, M. - Radiographic aspects of ruminants' bezoares. Clinical report in goats (Capra hircus). Rev. Fac. Med. vet. Zootec. Univ. S. Paulo, 12:127-32, 1975.

SUMMARY: Bezoares were clinically and radiographically detected in an 11 years old goat. Bezoares obtained from other animals were radiographically studied and the results compared to those observed in the clinical case, there reported.

The possible genesis of these structures is discussed.

UNITERMS: Ruminant*; Radiology*; Bezoar*.

\section{REFERENCIAS BIBLIOGRAFICAS}

1. BerguI, U. - Sulle lesioni provocate da corpi estranei d'origine animale e vegetale (egagropili) nei prestomaci dei bovini e segnatamente dei giovani animal. Progresso Vet., 11:276-9, 1956.

2. FERRARI, A. - Egagropili e Meteorismo ricorrente nel vitello. Clinica Vet., 80: 397-99, 1957.

3. HUTYRA, F. et al. - Patologia y terapeutica especiales de log animales domesticos. Barcelona, Editorial Labor, 1968. p. 71-73.
4. JUBB, K. V. F. \& KENNEDY, P. C. Pathologia de los animales domesticos. Barcelona, Editorial Labor, 1974. v. 2 p. 62 .

5. SEREN, E. - Enfermedadeg de los estomagog de log bovinos. Zaragoza, Acribia, 1975. p. 202-7.

6. ZANNELLI, C. \& ZIMBARO, F. S. - Su alcuni reperti di Bezoari nei bovini. Acta Med. Vet., 7:191-200, 1961.

Recebido para publicaçāo em 29-8-75

Aprovado para publicaçāo em 17-9.75 